\title{
Pérdida permisible de grasa en liposucción: :órmula y aplicación informática para cuantifictear un nuevo concepto
}

\section{Permissible loss of fat in liposuction: formula and computer application to quantify a new concept}

\author{
Raúl MANZANEDA CIPRIANI*, Fiorella Doris CANO GUERRA* \\ Gerardo A. ADRIANZEN NÚÑEZ**
}

\section{Resumen}

Introducción y objetivo. Existe poca evidencia científica de cuánta grasa se debería retirar en una lipoescultura, sólo parámetros rígidos recomendados. El concepto actual del volumen de grasa a retirar no puede ser generalizado, pues cada paciente tiene características intrínsecas distintas, por lo cual, el cálculo del volumen aspirado de grasa debería individualizarse para cada paciente.

El objetivo de este trabajo es crear una fórmula matemática predictiva para conocer la máxima cantidad de grasa a aspirar en una liposucción (pérdida permisible de grasa) en función de un volumen permisible sanguíneo seguro y de la edad ( $\sin$ necesidad de transfusiones y previniendo complicaciones hemodinámicas).

Material y método. Estudio cuantitativo, analítico, retrospectivo y de diseño no experimental. La población de estudio estuvo formada por pacientes operados de liposucción por un mismo cirujano en 3 clínicas privadas de Lima (Perú). Determinamos el tamaño mínimo de muestra con un nivel de confianza del $95 \%$ y margen de error del 5\%; la muestra final fue de 102 pacientes. Empleamos una base de datos en Microsoft Excel v19.00 para recolección de datos y técnicas descriptivas para homogeneizarlos. Luego, análisis multivariado teniendo en cuenta además edad del paciente y riesgo quirúrgico. Realizamos el análisis estadístico con el programa SPSS versión 25

Resultados. Contamos con 97 mujeres entre 18 a 54 años y 5 hombres entre 23 a 48 años (media de 32.5 años); el índice de masa corporal promedio (IMC) fue 22.06 .

El estudio de regresión lineal para las variables aspirado total de grasa y volumen permisible sanguíneo obtuvo un coeficiente de determinación muy importante $(\mathrm{R} 2=47 \%$ ) (fuerte asociación de predecibilidad). Dado este resultado, ampliamos el estudio a uno multivariable con las variables edad y riesgo quirúrgico, donde no determinamos asociación con respecto a riesgo quirúrgico, sin embargo, para edad la asociación se volvió más fuerte $(\mathrm{R} 2=49 \%)$, permitiendo construir una fórmula predictiva para la asociación de estas 3 variables y una aplicación informática para celulares y tabletas capaz de mejorar la aplicación de esta relación en la práctica clínica.

Conclusiones. El presente estudio determinó que las variables aspirado total de grasa y pérdida permisible de sangre son predictoras de la pérdida permisible de grasa. Así mismo, el análisis multivariable permitio aumentar el poder predictivo de esta relación al incluir la variable edad. De esta forma desarrollamos un método estadístico que permite determinar estas predicciones (fórmula) a fin de ayudar a los cirujanos a una mejor planificación quirúrgica de la lipoescultura. Para facilitar su uso, desarrollamos además una aplicación móvil gratuita que permite este cálculo de manera rápida y sencilla

\begin{tabular}{|c|c|c|}
\hline Palabras clave & $\begin{array}{l}\text { Lipoaspira } \\
\text { Liposucció } \\
\text { de grasa. }\end{array}$ & $\begin{array}{l}\text { en lipoaspirado, } \\
\text { permisible }\end{array}$ \\
\hline \multicolumn{2}{|c|}{ Nivel de evidencia científica } & 4b Diagnóstico \\
\hline \multicolumn{2}{|c|}{ Recihido [esta versión] } & 21 octubre/2020 \\
\hline \multicolumn{2}{|c|}{ Aceptado } & 22 enero/2021 \\
\hline
\end{tabular}

Background and objective. There is little scientific evidence of how much fat should be removed in a liposculpture, only rigid recommended parameters. The current concept of the volume of fat that should be removed cannot be generalized, since each patient has different intrinsic characteristics, therefore, the calculation of the aspirated volume of fat should be individualized for each patient.

The objective of this work is to create a predictive mathematical formula that allows us to know what is the maximum amount of fat that can be aspirated in liposuction (allowable fat loss) based on a safe allowable blood volume and age (without the need for blood transfusions and preventing hemodynamic complications).

Methods. A quantitative, analytical, retrospective study that responds to a non-experimental design was conducted. The study population was made up of patients undergoing liposuction by the same surgeon in 3 private clinics in Lima (Peru). The minimum sample size was determined with a confidence level of $95 \%$ database in Microsoft Excel v19.00 was used for data collection, likewise descriptive techniques were used to homogenize them. Then, the multivariate analysis was carried out, taking into account the patient's age and surgical risk. Statistical analysis was performed with the SPSS version 25 statistical program.

Results. We collected 97 women between 18 to 54 years old and 5 men between 23 to 48 years old (average age 32.5 years); the average body mass index (BMI) was 22.06

A linear regression study was carried out for the variables total fat aspirate and permissible blood volume, obtaining a very important coefficient of determination (R2 $=47 \%$ ) (strong association of predictability). Given this result, it was decided to extend the study to a multivariate one, with the variables age and surgical risk, where no association was determined with respect to surgical risk, however for age the association became stronger $(\mathrm{R} 2=49 \%)$, allowing the construction of a predictive formula for the association of these 3 variables, creating a computer application for cell phones and tablets to improve the application of this relationship in clinical practice.

Conclusion. The present study determined that the variables total fat aspiration and permissible blood loss are predictive variables of permissible fat loss. Likewise, the multivariate analysis allowed us to increase the predictive power of this relationship, as the age variable was included. In this way, it is possible to develop a statistical method that allows us to determine these predictions (formula), which will help surgeons to have a better surgical planning of liposculpture. To make this easier, a free mobile application was developed that allows this calculation quickly and easily

\begin{tabular}{lr} 
Key words & $\begin{array}{l}\text { Lipoaspirate, Lipoaspirate volume, } \\
\text { Liposuction, Permissible fat loss. }\end{array}$ \\
$\begin{array}{l}\text { Level of evidence } \\
\text { Received [this version] } \\
\text { Accepted }\end{array}$ & $4 b$ Diagnostic \\
\hline
\end{tabular}

Conflicto de intereses: Los autores declaran no tener ningún interés financiero relacionado con el contenido de este artículo. Financiación: No hubo fuentes externas de financiación para este trabajo. 
Introducción

Cuando se planteó la liposucción como cirugía de contorno corporal su objetivo principal fue retirar depósitos de grasa localizados, siendo esto suficiente para cumplir con los estándares de belleza de la época. ${ }^{(1)}$ Con el mayor conocimiento de la fisiología de la liposucción se han podido llegar a realizar liposucciones que abarcan más zonas, logrando un cambio mayor en el contorno corporal, evolucionando la técnica de liposucciones localizadas a lipoesculturas de contorno corporal total, siempre teniendo como concepto previo que la liposucción no es un tratamiento estándar para el paciente obeso. ${ }^{(2)}$

La revisión de la literatura científica muestra, de manera estandarizada, volúmenes estrictos como rangos de seguridad ${ }^{(3-7)}$ en relación al aspirado total de grasa, siendo las liposucciones mayores de $5000 \mathrm{cc}$ las cirugías que se asocian a mayor riesgo de complicaciones, ${ }^{(8-10)}$ por lo cual se propone que las cirugías que realicen lipoaspiraciones mayores de $5000 \mathrm{cc}$ se practiquen en centros de salud con unidad de cuidados intensivos acreditada. En Estados Unidos, algunos estados ${ }^{(2)}$ han impuesto restricciones relativas al volumen de aspiración, con límites que varían de 1000 a $5000 \mathrm{cc}$. El riesgo de complicaciones puede ser mayor a medida que aumenta el volumen de aspirado y el número de sitios anatómicos tratados. ${ }^{(8,9)}$ Están reportados fallecimientos ocasionales en pacientes sometidos a liposucción de gran volumen. ${ }^{(8,10)}$

El parámetro aislado más importante para conocer cuánta grasa podemos retirar en una liposucción es saber qué pérdida sanguínea vamos a tener en nuestro aspirado total. ${ }^{(10,11)}$ En la literatura existe una clasificación de liposucción de acuerdo a la cantidad de infiltración ${ }^{(12-17)}$ con su consecuente pérdida sanguínea expresada en porcentajes; es así, que la técnica seca se realiza sin infiltración y conlleva una pérdida sanguínea del 20 al $45 \%$ del aspirado total. La técnica húmeda, descrita por Illouz, ${ }^{(13,14)}$ implica la inyección de 200 a $300 \mathrm{ml}$ de infiltrado, teniendo una pérdida sanguínea del 4 al $5 \%$ del aspirado total. La técnica súperhúmeda, descrita por Fodor en 1986, ${ }^{(13,14)}$ utiliza una relación infiltrado-aspirado $1 / 1$ y la pérdida sanguínea es del 1 al $2 \%$ del volumen aspirado. La técnica tumescente introducida en 1985 por Klein (14), utiliza una relación infiltrado-aspirado 2-3/1 y la pérdida estimada de sangre que conlleva es del $1 \%$ del aspirado.

Los valores de pérdida sanguínea, de acuerdo al tipo de infiltración, no toman en cuenta la mayor pérdida sanguínea que se produce en la formación del tercer espacio en la zona intersticial, que representa el $98 \%$ de la pérdida sanguínea total. Esto es equivalente a perder la sangre de manera externa, porque la pérdida sanguínea en el tercer espacio no es reversible ni esa hemoglobina es funcional. ${ }^{(15)} \mathrm{El}$ conocimiento de la pérdida sanguínea real puede llevar al cirujano a realizar una liposucción segura.

El objetivo de este estudio es poner a disposición una manera individualizada y sencilla de estimar el volumen de grasa que podríamos extraer en una liposucción (pérdida permisible de grasa), sin aumentar el riesgo de complicaciones o precisar transfusiones sanguíneas, que podrían ser necesarias cuando la hemoglobina posquirúrgica llega a niveles menores de $10 \mathrm{~g} / \mathrm{dl}$; todo ello mediante un modelo predictivo adaptado en una aplicación informática gratuita que hemos llamado Pérdidas Permisibles de Grasa, para usar en dispositivos móviles.

\section{Material y método}

La presente investigación es un estudio cuantitativo, analítico, retrospectivo y responde a un diseño no experimental.

Para la recolección de datos revisamos 140 historias clínicas de pacientes operados en 3 clínicas privadas por un mismo cirujano en Lima (Perú), entre los años 2015 y 2017. Los criterios de inclusión tomados en cuenta fueron: a) anestesia general o epidural con sedación; b) hemoglobina posoperatoria igual o cercana a $10 \mathrm{~g} / \mathrm{dl}$ $(10 \pm 5)$; c) infiltración súperhúmeda (por cada litro de ClNa 9\% añadimos $1 \mathrm{ml}$ de epinefrina 1:1000, $20 \mathrm{ml}$ de lidocaína al 2\%); d) ausencia de complicaciones mayores posliposucción (necesidad de transfusión sanguínea, tromboembolismo pulmonar, embolia grasa, edema agudo de pulmón, muerte); e) parámetros de reposición de líquidos de acuerdo a los descritos por Rohrich y col., ${ }^{(16)}$ que nos ayudan a determinar el volumen de fluidos a dosificar de acuerdo al volumen aspirado en una liposucción y se determina de la siguiente forma: ratio de fluido intraoperatorio $=$ (volumen intravenoso en sala de operaciones + volumen de infiltración súper húmeda) / volumen de aspirado total de grasa. En este estudio se indica que la ratio es de 1.8 cuando el aspirado total es menor de 5000 cc y 1.2 cuando es mayor o igual a $5001 \mathrm{cc}$; de esta forma Rohrich y col. mencionan que, siguiendo estos parámetros de manejo, se previene la sobrecarga hídrica con sus respectivas complicaciones.

La selección de los casos para el estudio la hicimos entre los pacientes que acudieron a consulta externa para realizarse una lipoescultura como única opción quirúrgica (descartamos procedimientos combinados como implantes mamarios, rinoplastias, abdominoplastia, etc.). Todos los casos seleccionados, tanto hombres como mujeres, cumplieron con los siguientes parámetros: índice de masa corporal (IMC) menor o igual a $30 \mathrm{~kg} / \mathrm{m}^{2}$, clasi- 
ficación de laxitud de piel abdominal según Mattarasso menor a II, no haber tenido liposucción abdominal previa y riesgo quirúrgico según índice de Goldman menor o igual a II; además tomamos en cuenta las metas reales y objetivos claros determinados por los mismos pacientes.

Durante la preparación de los procedimientos quirúrgicos adoptamos medidas de cirugía segura, además de controles posoperatorios de hemoglobina y hematocrito. Practicamos infiltración tipo superhúmeda con solución de Klein modificada con cánula tipo Klein de $4 \mathrm{~mm}$ de diámetro y $35 \mathrm{~cm}$ de longitud. Comenzamos por la liposucción de la espalda y luego de la parte anterior del cuerpo con cánulas tipo basket, mercedes y candy, de 3-4-5 mm de diámetro y 30-35 cm de longitud, con máquina de aspiración. No empleamos ningún tipo de asistencia para la liposucción (tipo laser, vaser, etc.).

Las variables principales del estudio fueron: volumen aspirado total de grasa expresado en cc y máxima pérdida sanguínea permisible en cc (MPSA), ${ }^{(17,18)}$ que permiten calcular cuánta sangre en cc hay que perder para llegar a un valor de hemoglobina determinado en $\mathrm{g} / \mathrm{dl}$ (hemoglobina mínima aceptable).

La MPSA se determina de la siguiente manera:

- Volumen sanguíneo del paciente :

Ecuación de Nadler ${ }^{(19)}$

$\left(0.006012 \times\right.$ altura $\left.^{3}\right) /(14.6 \times$ peso $)+604$ en hombres

$\left(0.005835 \mathrm{x}\right.$ altura $\left.^{3}\right) /(15 \mathrm{x}$ peso $)+183$ en mujeres

Existe una forma más reducida de calcular el volumen sanguíneo que es: en hombres $(75 \mathrm{ml} \mathrm{x} \mathrm{kg})$ y e mujeres $(65 \mathrm{ml} \mathrm{x} \mathrm{kg})$.

- Hemoglobina actual del paciente: este valor se obtiene de los exámenes de laboratorio previos a la cirugía.

- Hemoglobina mínima aceptable: este es un valor variable y depende del valor con el que el cirujano desea trabajar; sin embargo, para motivos de nuestro estudio, nosotros determinamos $10 \mathrm{~g} / \mathrm{dl}$ como valor aceptable e invariable (pretendiendo mayor seguridad).

- Peso en kilogramos antes de la cirugía.

$\operatorname{MPSA}(\mathrm{cc})=[(\mathrm{Hb}(\mathrm{g} / \mathrm{dl})$ actual del paciente $-\mathrm{Hb}(\mathrm{g} / \mathrm{dl})$ mínimo aceptable)] x (peso en $\mathrm{kg}$ ) x (volumen sanguíneo del paciente en $\mathrm{cc}$ ).

Consideramos como variables secundarias la edad, con un rango de 18 a 55 años; el riesgo quirúrgico cardiovascular según índice de Goldman como protocolo de cirugía segura antes de cada procedimiento y determinado por un médico cardiólogo certificado; la estancia hospitalaria; y el tiempo en sala de recuperación. Realizamos mediciones de las funciones vitales, monitori- zadas tanto en sala de recuperación como en el cuarto hospitalario.

Para la recolección de datos revisamos las historias clínicas de los pacientes atendidos en 3 clínicas privadas de la ciudad de Lima Metropolitana (Perú) entre los años 2015 y 2017, dado que fue una investigación retrospectiva; todas las intervenciones habían sido realizadas por el mismo especialista en Cirugía Plástica. Consignamos la información obtenida en una ficha de recolección de datos y la almacenamos en una base de datos en Microsoft Excel (v19.00). Puesto que los datos fueron obtenidos de las historias clínicas, respetamos la confidencialidad de la información de los pacientes por aspectos éticos.

Concluido el trabajo de campo, procesamos los datos en el paquete estadístico SPSS versión 25 donde realizamos la estimación del modelo con regresión lineal simple a un nivel de confiabilidad del 95\%, inicialmente considerando volumen aspirado y pérdida permisible de sangre. Luego, para mejorar la predicción, consideramos adicionar las variables edad y riesgo quirúrgico empleando el modelo de regresión lineal múltiple.

\section{Resultados}

Inicialmente tuvimos una base de datos de 140 pacientes. Revisamos la presencia de datos discordantes con el procedimiento de diagrama de cajas, muy utilizado en investigaciones recientes para el control de la calidad de datos. Eliminando datos discordantes que afectaban la relación entre las dos variables, la base de datos final quedó formada por 102 pacientes, de los cuales 97 fueron mujeres entre los 18 a 54 años de edad y 5 fueron hombres entre los 23 a 48 años (media de edad de 32.5 años). El IMC promedio fue 22.06 (Tabla I).

Tabla I. CARACTERÍSTICAS DE LOS PARTICIPANTES

\begin{tabular}{|c|c|}
\hline \multicolumn{2}{|c|}{ TABLA DEMOGRÁFICA } \\
\hline MASCULINO & $5(23-48)^{*}$ \\
\hline FEMENINO & $97(18-54)^{*}$ \\
\hline TOTAL & 102 \\
\hline IMC PROMEDIO & $22.06(15.67-29.14)$ \\
\hline EDAD PROMEDIO & $32.15(18-54)$ \\
\hline $\begin{array}{c}\text { SANGRE CORPORAL } \\
\text { PROMEDIO } \\
\end{array}$ & $4226.35(2800-7100)$ \\
\hline $\begin{array}{l}\text { PERDIDA PERMISIBLE DE } \\
\text { SANGRE PROMEDIO }\end{array}$ & $1039.79(221-2100)$ \\
\hline VOL ASPIRADO PROMEDIO & $3633.63(200-8000)$ \\
\hline \multicolumn{2}{|c|}{$\begin{array}{l}\text { TIPO DE ANESTESIA } \\
\end{array}$} \\
\hline EPIDURAL+SEDACIÓN & 98 \\
\hline GENERAL & 4 \\
\hline LOCAL & 0 \\
\hline
\end{tabular}

$I M C=$ Índice de masa corporal .

$*=$ Rangos de edades. 
Analizando el tiempo promedio de estancia en sala de recuperación fue de 2.05 horas y el promedio de estancia hospitalaria de 21.66 horas; observamos rangos de normalidad debido a que no hubo complicaciones (no hubo episodios registrados de hipotensión o taquicardia) (Tabla II).

Tabla II. Estancia media en sala de recuperación y estancia de hospitalización

\begin{tabular}{|l|c|c|}
\hline Estadísticos & $\begin{array}{c}\text { Estancia en sala de } \\
\text { recuperaciones }\end{array}$ & $\begin{array}{c}\text { Estancia en } \\
\text { hospitalaria }\end{array}$ \\
\hline Media & 2.05 & 21.66 \\
\hline DS & 0.24 & 4.06 \\
\hline
\end{tabular}

Cabe precisar que un requisito importante para utilizar el modelo de regresión es la presencia de normalidad en los datos y que la relación sea lineal. En caso contrario, si se da no linealidad, no es posible la construcción del modelo.

Para construir el modelo predictivo inicial utilizamos la prueba de regresión lineal simple a un nivel de confianza del 95\%, donde la variable dependiente era el volumen aspirado total de grasa $(\mathrm{Y})$ y la variable independiente la pérdida permisible de sangre $(\mathrm{X})$. El modelo final estableció un coeficiente de determinación (fuerza de predicción entre las dos variables) de regresión lineal para ambas variables de $\mathrm{R}^{2}=47 \%$. Este resultado nos indica que la variable volumen aspirado total de grasa $\mathrm{y}$ pérdida permisible de sangre son variables predictivas, que según normas estadísticas es muy alto, ya que supera el 30\% de variabilidad. ${ }^{(20)}$ (Tabla III y Gráfico 1). Es decir, ambas variables tienen asociación predictiva de alta fuerza estadística en base a que explican casi el $50 \%$ de la variabilidad de pérdida permisible de sangre con respecto al aspirado total de grasa.

Tabla III. Modelo predictivo simple.

\begin{tabular}{|l|c|c|c|c|}
\hline \multicolumn{7}{|c|}{ Resumen del modelo } \\
\hline Modelo & $\mathrm{R}$ & $\mathrm{R}$ cuadrado & $\begin{array}{c}\text { R cuadrado } \\
\text { ajustado }\end{array}$ & $\begin{array}{c}\text { Error estándar } \\
\text { de la } \\
\text { estimación }\end{array}$ \\
\hline 1 &, $686^{\mathrm{a}}$ & .471 & .466 & 1216.754 \\
\hline
\end{tabular}

Única variable: pérdida permisible de sangre

Por otro lado, al tener una asociación fuerte, buscamos otras variables que contribuyan al análisis predictivo, motivo por el cual optamos por un estudio multivariable en el que consideramos como variables adicionales la variable edad y la variable riesgo quirúrgico cardiovascular (Goldman), debido a que son parte de la evaluación inicial y preparación prequirúrgica de cada paciente.

Con respecto a la edad, es una variable que determina muchos cambios fisiológicos, determinados por los
Gráfico 1. Volumen de aspiración frente a Pérdida permisible de sangrey estancia de hospitalización

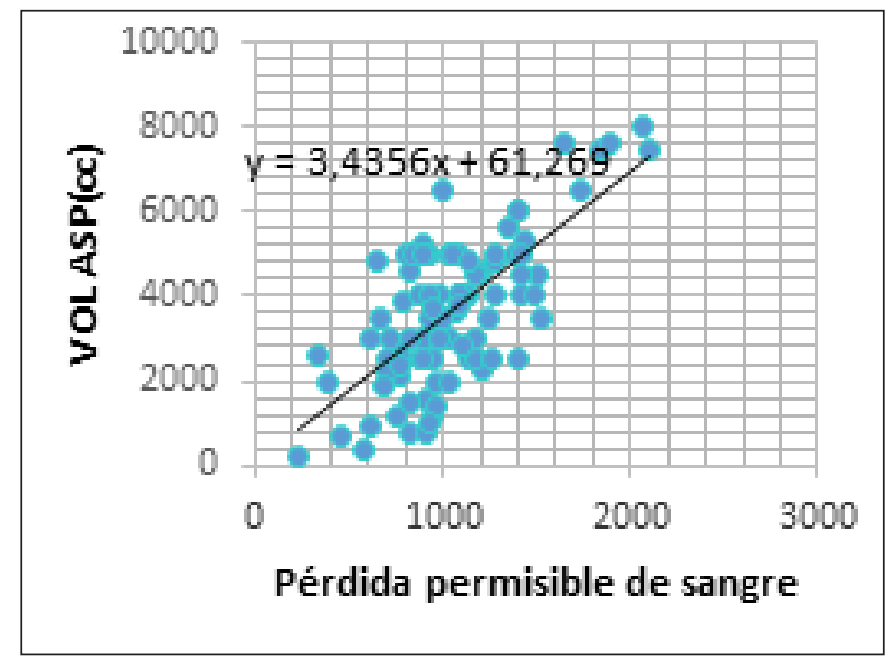

Se observa la relación lineal entre el volumen aspirado y la pérdida permisible de sangre.

cambios adaptativos estructurales de nuestro cuerpo; es así que a nivel cardiovascular la resistencia periférica va aumentando en base a un desbalance entre el efecto vasodilatador del óxido nítrico y vasoconstrictor de la angiotensina II, produciendo aumento de la presión arterial y disminuyendo la capacidad de adaptabilidad hemodinámica frente a una pérdida sanguínea aguda, motivo por el cual la edad se vuelve fundamental al momento de determinar la posibilidad de un efecto adverso con respecto al análisis de pérdida permisible de grasa y sangre en un acto operatorio como la liposucción. ${ }^{(21-23)}$

El valor exacto de hemoglobina al cual el gasto cardiaco aumenta, varía entre los individuos y está influenciado por la edad y también por si la anemia se desarrolla de manera aguda o lenta; en algunos pacientes el gasto cardíaco no aumenta hasta que la hemoglobina cae a 7 u $8 \mathrm{~g} / \mathrm{dl}$, motivo por el cual, a nuestro parecer, hemos creído conveniente priorizar la seguridad del paciente tomando como valor crítico la hemoglobina de $10 \mathrm{~g} / \mathrm{dl}$ que es la que usamos en la fórmula que planteamos en este trabajo. ${ }^{(24-27)}$

Por tal motivo realizamos el análisis multivariado, donde encontramos que la variable riesgo quirúrgico cardiovascular (Goldman) no tiene suficiente nivel de confianza para determinar asociación; sin embargo, la variable edad fue muy importante dado que elevó nuestro coeficiente de determinación en el nuevo análisis multivariado a $\mathrm{R}^{2} 49 \%$, demostrando que se puede incluir como variable de predicción de las variables pérdida permisible de sangre y aspirado total de grasa (pérdida permisible de grasa), esta última tomando la relevancia de poder asociar cuánta grasa podemos retirar para tener una pérdida de sangre específica, en una edad determinada y en una cirugía de liposucción (Tabla IV). 
Tabla IV. Resumen del modelo predictivo múltiple.

Resumen del modelo

\begin{tabular}{|l|r|r|r|r|}
\hline Modelo & \multicolumn{1}{|c|}{$\mathrm{R}$} & R cuadrado & $\begin{array}{l}\text { R cuadrado } \\
\text { ajustado }\end{array}$ & $\begin{array}{c}\text { Error estandar } \\
\text { de la estimación }\end{array}$ \\
\hline \hline & 17019 & .492 & .476 & 1204.399 \\
\hline
\end{tabular}

El modelo predictivo múltiple final (predictores: pérdida permisible de sangre, edad y riesgo quirúrgico) quedaría establecido de la siguiente manera:

$Y 2=383.725+3.406$ (perdida permisible sangre) - 29.116 (edad); donde "Y2" es igual a pérdida permisible de grasa.

\section{Discusión}

Existe en la actualidad poca evidencia científica sobre cuánta grasa se debería retirar en una lipoescultura, ${ }^{(24,25)}$ sólo parámetros rígidos recomendados. El concepto actual del volumen de grasa que se debe retirar no puede ser generalizado, pues cada paciente tiene características intrínsecas distintas, por lo cual, el cálculo del volumen aspirado de grasa debe ser individualizado para cada paciente. En busca de esta premisa, algunos cirujanos han realizado estudios para llegar a este concepto. Chow, en $2015,{ }^{(25)}$ presenta un estudio para cuantificar el riesgo integral asociado a diferentes volúmenes y su IMC. El resultado demuestra que volúmenes superiores a $100 \mathrm{cc}$ por unidad de masa corporal aumentan el riesgo de complicaciones; en este estudio evalúan complicaciones leves y graves, siendo las de mayor frecuencia seromas y retracciones de piel. En contrapartida, Swanson ${ }^{(7)}$ realizó una revisión prospectiva con una tasa de inclusión del $100 \%$, en la que todos los participantes fueron tratados por el mismo cirujano, con técnica de infiltración súperhúmeda y eliminando el sesgo de selección y factores de confusión. El análisis se hizo mediante correlación de Pearson, demostrando que no existía correlación entre el volumen de aspiración y la frecuencia de complicaciones; asimismo, el IMC no era un factor de riesgo y concluyendo que, en promedio, un volumen de aspiración de 5000 cc se correlaciona con una pérdida de sangre de 1.000 cc, de tal forma que si el cirujano decide aspirar más de $5000 \mathrm{cc}$ de grasa, debería tomar precauciones por posibles eventos adversos. Swanson manifestó también que si un cirujano excede el rango de seguridad definido por algunos autores de $5000 \mathrm{cc}^{(23,24)}$ podría ser injustamente culpado y la liposucción se podría volver prohibitiva.

La pérdida sanguínea basada en porcentajes de acuerdo al tipo de infiltración ${ }^{(12,14)}$ es un parámetro adoctrinado en algún momento de nuestra educación, fundamentado en la medición del lipocrito sin tener en cuenta algunos otros factores fisiológicos de mayor relevancia, siendo el lipocrito, a nuestro parecer, ${ }^{(12-15)}$ poco preciso en relación a la pérdida sanguínea real observada en la práctica habitual. Podría existir una naturaleza engañosa de estimar la pérdida sanguínea en base al lipocrito, por los niveles insignificantes de hematocrito en el aspirado total que representan solo el 2\% de la pérdida sanguínea experimentada en una liposucción; existe una pérdida de sangre que se encuentra en el espacio intersticial (tercer espacio) que representa el $98 \%{ }^{(21)}$ de la pérdida sanguínea en la liposucción. Este tercer espacio no es tomado en cuenta cuando se realiza la clasificación de liposucción por el tipo de infiltrado; realizando un cálculo aproximado, si infiltramos $5000 \mathrm{cc}$ y retiramos $5,000 \mathrm{cc}$, tendríamos una liposucción tipo súperhúmeda en la que la pérdida sanguínea vendría a ser de 50 cc (1\%) y tendría una repercusión mínima en el estado hemodinámico y en la hemoglobina posoperatoria.

Como hemos mencionado anteriormente, la pérdida permisible de sangre (en cc) es una variable que se determina de acuerdo a una fórmula muy utilizada en anestesiología y que se estable de acuerdo a valores de hemoglobina, peso y volumen sanguíneo del paciente, con el significado de cuánta sangre podemos perder durante un acto quirúrgico para llegar a una hemoglobina determinada. ${ }^{(17-19)}$

El volumen aspirado total de grasa (en cc) se obtiene de la lipoaspiración corporal y está formado por grasa, sangre y solución súperhúmeda; así mismo, la cantidad obtenida depende exclusivamente del cirujano.

Durante el análisis estadístico de nuestra base datos, obtuvimos que nuestras variables: aspirado total de grasa $(\mathrm{Y})$ y volumen permisible sanguíneo $(\mathrm{X})$ tuvieron un coeficiente de determinación muy importante $\left(\mathrm{R}^{2}=47 \%\right)$; esto quiere decir que tenemos una correlación lineal entre ambas variables con una fuerza de gran magnitud. De esta forma se planteó la siguiente relación: $\mathrm{Y}=3.4356 \mathrm{X}+$ 61.269 (Gráfico 1), es decir, tuvimos una correlación con alta predicción. Basándonos en esto, decidimos ampliar nuestro análisis estadístico a uno multivariable, incluyendo la edad y el riesgo quirúrgico en este proceso y obteniendo que la variable edad aumenta considerablemente el coeficiente de determinación $\left(\mathrm{R}^{2}=49 \%\right)$ (Tabla IV), sin embargo la variable riesgo quirúrgico no mostró ser significativa $(\mathrm{p}>0.05)$.

En el análisis multivariable obtuvimos la siguiente relación: $\mathrm{Y}_{2}=383.725+3.406$ (pérdida permisible sangre) - 29.116 (edad), es decir, que ahora podemos utilizar esta fórmula para poder predecir cuál es la máxima cantidad de grasa que se puede aspirar para llevar la hemoglobina del paciente a $10 \mathrm{~g} / \mathrm{dl}$, es decir, la pérdida permisible de grasa $\left(\mathrm{Y}_{2}\right)$.

Durante el estudio no se presentaron complicaciones mayores (necesidad de transfusión sanguínea, tromboembolismo pulmonar (TEP), embolia grasa, edema agudo de pulmón y muerte). 
Dando un ejemplo con la fórmula predictiva que realizamos (pérdida permisible de grasa) en un paciente de 30 años con $70 \mathrm{~kg}$ de peso, $\mathrm{Hb}$ de $13 \mathrm{~g} / \mathrm{dl}$ y una $\mathrm{Hb}$ mínima posoperatoria de $10 \mathrm{~g} / \mathrm{dl}$, teniendo una pérdida permisible de sangre de $1212 \mathrm{cc}$, y aplicando nuestra fórmula predictiva de pérdida permisible de grasa, se podría obtener un volumen de aspiración de 3638 cc aproximadamente sin la necesidad de transfusiones sanguíneas.

Lo que nos motivó a realizar el presente trabajo fue los límites estrictos que existen con respecto al volumen de lipoaspiración segura, determinada por reuniones científicas, estados de algunos países y sociedades de cirujanos plásticos, sin tener en cuenta la individualidad de cada paciente. Cabe resaltar que no estamos a favor de practicar megaliposucciones.

La liposucción de grandes volúmenes, definida como aquella superior a $5000 \mathrm{cc}$ de grasa aspirada, tiene como finalidad mejorar el contorno corporal de la persona que lo necesite, siendo en la mayoría de los casos pacientes con sobrepeso y obesidad. Teniendo en cuenta que la liposucción no es una cirugía para bajar de peso, ${ }^{(26,27)}$ existen sin embargo algunos estudios que sugieren una mejora en los riesgos cardiovasculares y metabólicos después de la

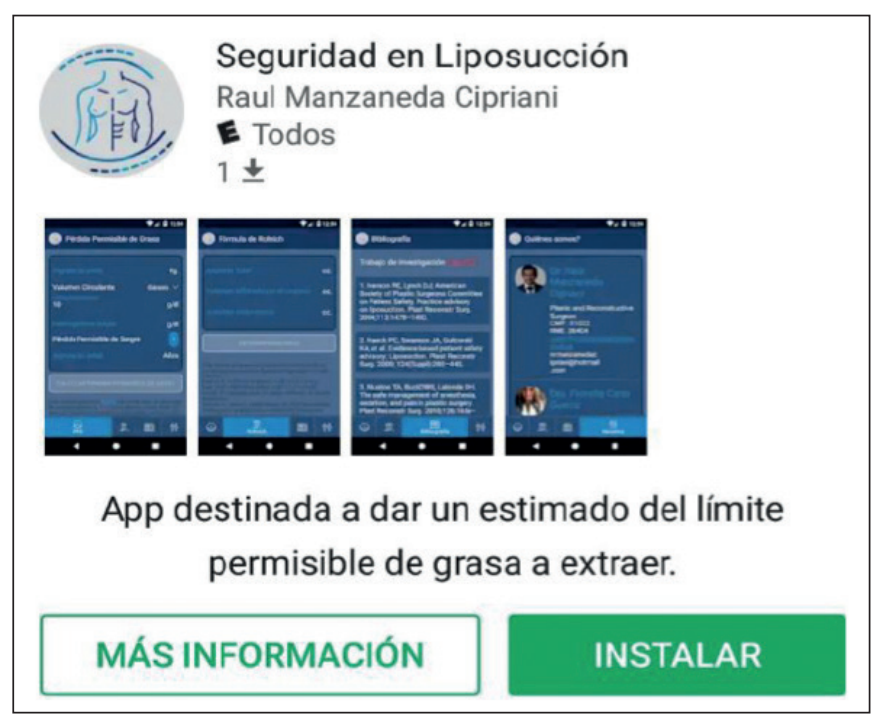

Figura 1. Aplicación disponible en Android.

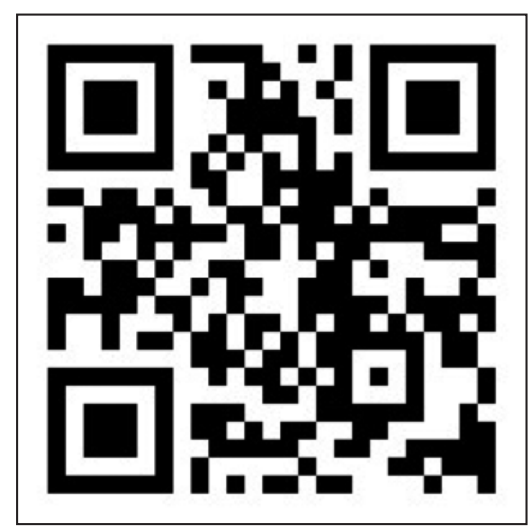

Figura 2. Código QR para dispositivos Android. liposucción, ${ }^{(28)}$ pero también es cierto que existen riesgos inherentes a estos pacientes como son las alteraciones en la cicatrización y el aumento de riesgo de sufrir infecciones. Por lo tanto, a nuestro parecer, la liposucción no debe ser considerada como un tratamiento para la obesidad y si se debe elegir entre una megaliposucción con sus posibles beneficios, en contraposición a sus posibles complicaciones, prefiriendo siempre un paciente con el menor riesgo posible ante una mejora estética.

Con respecto al manejo hidroelectrolítico en el intraoperatorio, nos guiamos por el trabajo de Rohrich y col. ${ }^{(16)}$ cuya fórmula hemos mencionado anteriormente. A nuestro parecer, estos parámetros de resucitación podrían inclinarse en algunos casos hacia la sobrecarga, por lo que sugerimos el manejo personalizado ya que la tendencia actual es usar la menor cantidad de líquido intravenoso en el contexto clínico de una liposucción.

Decidimos convertir la fórmula de pérdida permisible de grasa y la fórmula de Rohrich en una aplicación para celulares y tabletas, ya que en el día a día quirúrgico se torna tedioso realizar cálculos matemáticos. En esta aplicación se pueden colocar los datos de manera sencilla y así tener a mano cálculos aproximados de cuánta grasa se podría retirar, a la vez que una ayuda para el anestesiólogo con respecto al manejo hidroelectrolítico intraoperatorio. Esta aplicación se mantiene en constante actualización, de forma que la que se encuentra en línea

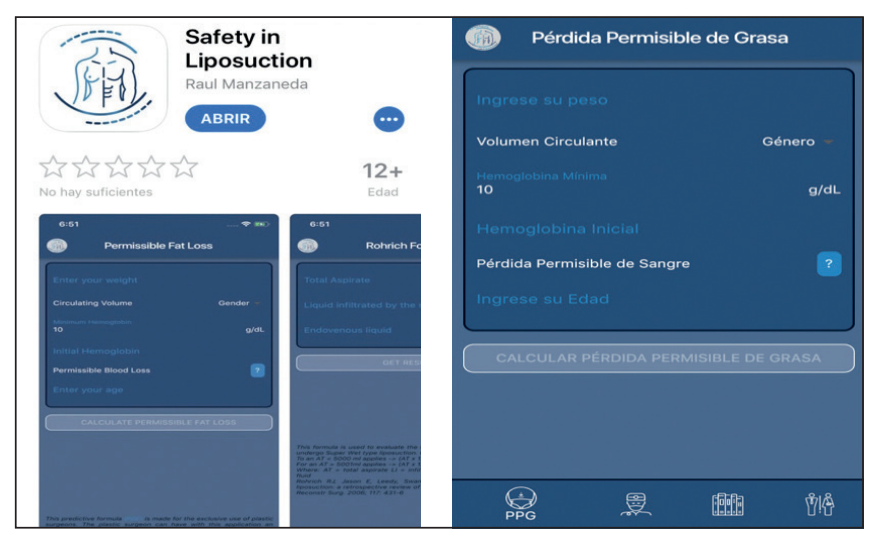

Figura 3: Aplicación disponible en iOS

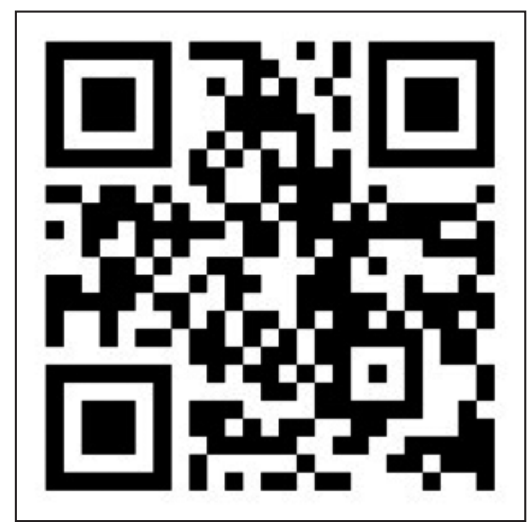

Figura 4. Código QR para dispositivos iOS 
en el momento de publicar este artículo es la segunda versión. Se ha desarrollado con un ingeniero informático y está registrada a nombre de los autores con licencia para android e iOs.

Link de aplicativo disponible en android demanera gratuita: https://play.google.com/store/apps/details?id=com. raul.manzaneda.seguridadenliposuccion2. (Fig. 1 y 2), y en iOS https://itunes.apple.com/us/app/safety-in-liposuction/id1446493571 (Fig. 3 y 4). En esta aplicación se puede encontrar la fórmula de pérdida permisible de grasa, asi como la fórmula de reposición de líquidos en base a la publicación de Rohrich y col. ${ }^{(16)}$

\section{Conclusiones}

Demostramos estadísticamente que la pérdida permisible de sangre es un predictor del volumen aspirado total de grasa, y que por lo tanto es posible construir un modelo predictivo a un nivel de confianza del $95 \%$.

Adicionalmente, la variable edad, al ser sometida en el análisis multivariado, brinda mayor poder estadístico a la correlación de las variables aspirado de grasa total y pérdida permisible de sangre, con un $95 \%$ de confianza. Esto puede ser un factor relevante, ya que la edad es directamente proporcional a la posibilidad de tener un evento adverso en una cirugía.

El análisis multivariado de edad y pérdida permisible de sangre permite calcular con una buena predicción, de acuerdo a la significancia de su correlación, los niveles permisibles de pérdida de grasa con un nivel de confianza del 95\%.

El uso de una aplicación móvil que permite calcular el manejo hidroelectrolítico intraoperatorio y la pérdida permisible de grasa, en el contexto de una cirugía de liposucción, nos ayuda a tener cirugías con mayores índices de seguridad y mejores protocolos de preparación quirúrgica de los pacientes.

\section{Dirección del autor}

Dr. Raúl Martin Manzaneda Cipriani

Aurelio Miro Quesada 1048

San isidro, Lima, Perú

Correo electrónico: rmanzanedacipriani@hotmail.com

\section{Bibliografía}

1. Avelar J. Regional Distribution and Behavior of the Subcutaneous Tissue Concerning Selection and Indication for Liposuction. Aesth Surg J. 1989;13:155-165.

2. Haeck P, Swanson J, Gutowski K, Basu CB, Wande A. et al. The ASPS Patient Safety Committee Evidence-Based Patient Safety Advisory: Liposuction. Plast Rec Surg. 2009; 124(4S): 28S-44S.
3. Iverson RE, Lynch DJ. American Society of Plastic Surgeons Committee on Patient Safety. Practice advisory on liposuction. Plast Reconstr Surg. 2004;113:1478-1490.

4. Haeck PC, Swanson JA, Gutowski KA, et al. Evidence-based patient safety advisory: Liposuction. Plast Reconstr Surg. 2009;124(Suppl):28S-44S.

5. Mustoe TA, Buck DW II, Lalonde DH. The safe management of anesthesia, sedation, and pain in plastic surgery. Plast Reconstr Surg. 2010;126:165e-176e.

6. Brown SA, Lipschitz AH, Kenkel JM, et al. Pharmacokinetics and safety of epinephrine use in liposuction. Plast Reconstr Surg. 2004;114:756-763; discussion 764-765.

7. Swanson E. Prospective clinical study reveals significant reduction in triglyceride level and white blood cell count after liposuction and abdominoplasty and no change in cholesterol levels. Plast Reconstr Surg. 2011;128:182e-197e.

8. Pitman GH, Aker JS, Tripp ZD. Tumescent liposuction: A surgeon's perspective. Clin Plast Surg. 1996;23:633-641; discussion 642-645.

9. Talmor M, Fahey TJ II, Wise J, Hoffman LA, Barie PS. Large-volume liposuction complicated by retroperitoneal hemorrhage: Management principles and implications for the quality improvement process. Plast Reconstr Surg. 2000;105:2244-2248; discussion 2249-2250.

10. Gross JB. Estimating allowable blood loss: Corrected for dilution. Anesthesiology 1983;58:277-280.

11. Matarasso A. Lidocaine in ultrasound-assisted lipoplasty. Clin Plast Surg. 1999;26:431-439.

12. Grazer FM. Quantitative analysis of blood and fat in suction lipectomy aspirates (Discussion). Plast Reconstr Surg. 1986; 78:770-772.

13. Samdal F, Amland PF, Bugge JF. Plasma lidocaine levels during suction-assisted lipectomy using large doses of dilute lidocaine with epinephrine. Plast Reconstr Surg. 1994;93:12171223.

14. Rubin JP, Bierman C, Rosow CE, et al. The tumescent technique: The effect of high tissue pressure and dilute epinephrine on absorption of lidocaine. Plast Reconstr Surg. 1999;103:990-996; discussion 997-1002.

15. Karmo FR, Milan MF, Silbergleit A. Blood loss in major liposuction procedures: A comparison study using suctionassisted versus ultrasonically assisted lipoplasty. Plast Reconstr Surg. 2001;108:241-247; discussion 248-249.

16. Rohrich RJ, Jason E, Leedy, Swamy JR. Fluid resuscitation in liposuction: a retrospective review of 89 consecutive patients. Plast Reconstr Surg. 2006;117:431-436.

17. Bourke DL, Smith TC. Estimating allowable hemodilution. Anesthesiology. 1974;41(6):609-612.

18. Feldman JM, Roth JV, Bjoraker DG. Maximum blood savings by acute normovolemic hemodilution. Anesth Analg. 1995;80(1):108-113.

19. Nadler SB, Hidalgo JU, Bloch T. Prediction of blood volume in normal human adults. Surgery.1962; 51(2):224-232.

20. Lizaraso F, Medina J. Fundamentos de Estadística Médica. Fondo Editorial USMP. Lima-Peru,2013, Pp.171-175.

21. Stauder R, Valent $\mathbf{P}$, Theurl I. Anemia at older age: etiologies, clinical implications, and management. Blood. 2018;131(5):505514.

22. Forman D, Fleg J, Wenger N. Aging and Specific Cardiovascular Conditions. En Zipes D, Libby P, Bonow R, Mann D, Tomase1 li G. Braunwald s Heart Disease. 11th ed. 2019. Pp. 4396-4470.

23. Rohrich RJ, Leedy JE, Swamy R, Brown SA, Coleman J. Fluid resuscitation in liposuction: A retrospective review of 89 con- secutive patients. Plast Reconstr Surg. 2006;117:431-435.

24. Giese SY, Neborsky R, Bulan E, Spear SL, Yanovski JA. Improvements in cardiovascular risk profile after large-volume lipoplasty: A 1-year follow-up study. Aesth Surg J. 2001;21:527531. 
25. Chow I, Alghoul MS, Hanwright PJ, et al. Is there a safe lipoaspirate volume? A risk assessment model of liposuction volume as a function of body mass index. Plast Reconstr Surg. 2015; 136:474-483.

26. Chia CT, Neinstein RM, Theodorou SJ. Evidence-Based Medicine: Liposuction. Plast Reconstr Surg. 2017;139(1):267e-274e.
27. Khanna A, Filobbos G. Avoiding unfavourable outcomes in liposuction. Indian J Plast Surg. 2013;46(2):393-400.

28. De Jong RH. Body mass index: Risk predictor for cosmetic day surgery. Plast Reconstr Surg. 2001;108:556-561; discussion 562-563.

\section{Comentario al artículo "Pérdida permisible de grasa en liposucción: fómula y aplicación informática para cuantificar un nuevo concepto"}

\section{Ernesto A. MORETTI}

Cirujano Plástico. Jefe del Servicio de Cirugía Plástica del Hospital Privado Rosario. Titular de la Cátedra de Anatomía Humana, Facultad de Medicina, UAI, Rosario, Argentina.

Director de la Fundación Docente FILACP (2020-2022).

Con atención he leído el trabajo de los doctores Manzaneda Cipriani, Cano Guerra y Adrianzen Núñez. Es un trabajo de investigación cuantitativo, analítico y retrospectivo sobre seguridad de los volúmenes a retirar en las liposucciones. Se analizaron datos obtenidos en pacientes con liposucción tradicional con infiltración de las zonas quirúrgicas con técnica superhúmeda.

Todos conocemos lo tedioso y dificultoso que se torna para los cirujanos plásticos realizar cálculos matemáticos y estadísticos. Los autores lo demuestran en Material y método, donde relatan una inumerable cantidad de fórmulas y análisis realizados en 102 pacientes.

Lo que resulta novedoso e ingenioso es la aplicación para IOS y ANDROID que presentan los autores. Dicha aplicación permite cargar datos de forma rápida y sencilla, logrando obtener valores certeros de cuánta grasa se puede retirar, a la vez que brinda una ayuda para el anestesiólogo con respecto al manejo hidroelectrolítico intraoperatorio.

Esta aplicación fue realizada por un ingeniero informático en forma conjunta con los autores que proveyeron los datos a colocar:
1- Ingreso del peso

2- Género (variación importante si es hombre o mujer)

3- Hemoglobina inicial

4- Edad

Automáticamente, y en forma inmediata, se obtiene la Pérdida Permisible de Sangre así como la Pérdida Permisible de Grasa en centímetros cúbicos.

La aplicación se puede bajar de forma gratuita y personal. Yo ya la tengo en mi móvil. Me permitió en 5 segundos obtener un valor predictivo confiable (confianza 95\%) de cuánta grasa se puede aspirar en una liposucción tradicional con técnica superhúmeda. Tambien la aplicación posee la fórmula de Rohrich, en la cual el cirujano coloca el aspirado total en centímetros cúbicos, el volumen infiltrado por el cirujano y el volumen endovenoso aportado por el anestesiólogo. Se obtiene así otro valor de importancia para la reposición hídrica, ya que el resultado obtenido demuestra el déficit o exceso aportado.

Deseo felicitar a los autores por el excelente trabajo enviado a nuestra Revista y por la grandeza de compartir una aplicación disponible para cualquier móvil que permite en instantes conocer los valores máximos a retirar mediante liposucción para cada paciente, así como orientar el aporte hídrico a restituir. 


\section{Respuesta al comentario del Dr. E. A. Moretti}

\section{Raúl MANZANEDA CIPRIANI}

Estimado Dr. Moretti, agradecemos genuinamente su invaluable comentario y punto de vista acerca de nuestro trabajo. Como equipo estamos comprometidos a continuar realizando investigación y métodos que nos permitan tener una evaluación más óptima de nuestros pacientes.

La globalización, el acceso a internet y aplicativos móviles, han facilitado el poder compartir información y así mismo el poder acceder a herramientas útiles, que de forma rápida, nos ayuden a tener un mejor abordaje y manejo de nuestros pacientes.

La liposucción es una de las cirugías que más se realizan a nivel mundial, ${ }^{(1)}$ esto nos debe comprometer a mejorar la calidad y la seguridad de este procedimiento; así mismo, el estudio del paciente y la personalización de la cirugía es un hito importante durante la planificación quirúrgica, de esta forma el cálculo de la Pérdida Permisible de Sangre así como de la Pérdida Permisible de Grasa y la aplicación de la fórmula de Rohrich, permiten justamente dar ese toque de seguridad y personalización que planteamos.

Lo tedioso y complicado que configura el tener que realizar cálculos de diferentes variables para poder tener seguridad en cirugía, muchas veces nos hace caer en el campo de la aproximación, sobre todo cuando hablamos de volúmenes y rangos de seguridad como los que se describen en nuestro trabajo. De esta forma, el poder realizar cálculos de forma rápida y precisa que nos permitan tomar decisiones acerca de cómo manejar volúmenes en cirugía, se vuelve de gran relevancia, sobre todo para futuros trabajos que busquen el desarrollo de otras formulaciones que ayuden a mejorar la calidad y seguridad de nuestras cirugías.

El comentario que usted nos ha dirigido nos permite tener la seguridad de que el objetivo de nuestro trabajo ha sido reconocido de forma correcta, pues nuestra intención es ayudar a los cirujanos plásticos a tener mayor seguridad y eficacia para cálculos que normalmente se vuelven complicados y demandan mucho tiempo.

El aplicativo desarrollado es actualmente usado en la práctica quirúrgica diaria de nuestro equipo, con muy buenos resultados con respecto a evitar eventos adversos secundarios a la cirugía. Al momento, nuestro aplicativo ha tenido más de 100 descargas en ambas plataformas IOS y ANDROID y aunque aún no es muy reconocido, tenemos la seguridad de que pronto podrá tener la relevancia que nuestros objetivos se han trazado.

Agradecemos una vez más la oportunidad de poder mostrar nuestro trabajo; así mismo, reafirmamos nuestro compromiso de seguir trabajando en el desarrollo de herramientas que nos permitan mejorar la seguridad y calidad de nuestros procedimientos, con el fin de ofrecerle a nuestros pacientes nuestro mayor compromiso como profesionales.

\section{Bibliografía}

1. ISAPS international Survey on Aesthetic/Cosmetic Procedures Performed in 2019. Disponible en: https://www. isaps.org/wp-content/uploads/2020/12/ISAPS-Global-Survey-2019-Press-Release-Spanish.pdf 
